\title{
Characteristics of Indigenous primary health care service delivery models: a systematic scoping review
}

\author{
Stephen G. Harfield ${ }^{1,2,4^{*}}$ D, Carol Davy ${ }^{1,2}$, Alexa McArthur ${ }^{3}$, Zachary Munn ${ }^{3}$, Alex Brown ${ }^{1,4}$ and Ngiare Brown ${ }^{1,5}$
}

\begin{abstract}
Background: Indigenous populations have poorer health outcomes compared to their non-Indigenous counterparts. The evolution of Indigenous primary health care services arose from mainstream health services being unable to adequately meet the needs of Indigenous communities and Indigenous peoples often being excluded and marginalised from mainstream health services. Part of the solution has been to establish Indigenous specific primary health care services, for and managed by Indigenous peoples. There are a number of reasons why Indigenous primary health care services are more likely than mainstream services to improve the health of Indigenous communities. Their success is partly due to the fact that they often provide comprehensive programs that incorporate treatment and management, prevention and health promotion, as well as addressing the social determinants of health. However, there are gaps in the evidence base including the characteristics that contribute to the success of Indigenous primary health care services in providing comprehensive primary health care. This systematic scoping review aims to identify the characteristics of Indigenous primary health care service delivery models.
\end{abstract}

Method: This systematic scoping review was led by an Aboriginal researcher, using the Joanna Briggs Institute Scoping Review Methodology. All published peer-reviewed and grey literature indexed in PubMed, EBSCO CINAHL, Embase, Informit, Mednar, and Trove databases from September 1978 to May 2015 were reviewed for inclusion. Studies were included if they describe the characteristics of service delivery models implemented within an Indigenous primary health care service. Sixty-two studies met the inclusion criteria. Data were extracted and then thematically analysed to identify the characteristics of Indigenous PHC service delivery models.

Results: Culture was the most prominent characteristic underpinning all of the other seven characteristics which were identified - accessible health services, community participation, continuous quality improvement, culturally appropriate and skilled workforce, flexible approach to care, holistic health care, and self-determination and empowerment.

Conclusion: While the eight characteristics were clearly distinguishable within the review, the interdependence between each characteristic was also evident. These findings were used to develop a new Indigenous PHC Service Delivery Model, which clearly demonstrates some of the unique characteristics of Indigenous specific models.

Keywords: Primary health care, Models of care, Service delivery, Indigenous, Aboriginal and Torres Strait Islander, American Indian and Alaska Native

\footnotetext{
* Correspondence: s.harfield@ug.edu.au

${ }^{1}$ Wardliparingga Aboriginal Health Research Unit, South Australian Health

and Medical Research Institute, Adelaide, South Australia, Australia

${ }^{2}$ School of Public Health, Faculty of Health Sciences, The University of

Adelaide, Adelaide, South Australia, Australia

Full list of author information is available at the end of the article
} 


\section{Background}

Indigenous populations have poorer health outcomes compared to their non-Indigenous counterparts [1]. The experience of colonisation, and the long-term effects of being colonised, has caused inequalities in Indigenous health status, including physical, social, emotional, and mental health and wellbeing [2]. For example, in 2012 the gap in life expectancy between Aboriginal and Torres Strait Islander Australians and non-Indigenous Australians was 10 years [3]. Similar gaps in life expectancy exist in New Zealand [4], Canada [5] and the United States [6].

The evolution of Indigenous primary health care (PHC) services arose from the inability of mainstream health services to adequately meet the needs of Indigenous communities $[3,7,8]$. It was also a response to the reality that Indigenous peoples were often excluded and marginalised from mainstream health services [9]. Part of the solution has been to establish Indigenous specific PHC services, for and managed by Indigenous peoples.

In Australia, the first Aboriginal PHC service was established in 1971 [9] and there are now over 150 Aboriginal Community Controlled Health services across the country [10]. In New Zealand, health reform in the early 1990's led to the development of Māori health providers. This has resulted in a combination of national and locally controlled Māori led initiatives that are committed to improving Māori health [7]. In Canada, the enactment of the Health Transfer Policy in the late 1980's initiated the transfer of existing community-based and regional health services into First Nation and Inuit control [11, 12], and more recently the establishment of First Nations and Inuit Health Authorities [13]. In the United States, the provision of health services for American Indians and Alaska Natives began as early as the nineteenth Century and continued through the 1930's, 1950's and 1970's with a number of policy reforms, culminating in what is now known as the Indian Health Services [14-16].

There are a number of reasons why Indigenous PHC services are more likely than mainstream services to improve the health of Indigenous communities. One of the primary reasons is that Indigenous PHC services are often controlled by their local communities [7, 13, 14, 17] and therefore are underpinned by the values and principles of the communities they serve [18]. Their success is also due to the fact that they often provide comprehensive programs that incorporate treatment and management, prevention and health promotion, as well as addressing the social determinants of health [14].

Despite their success, there are gaps in the evidence base including the characteristics that contribute to the success of Indigenous PHC services in providing comprehensive PHC. This systematic scoping review sought to address this gap by identifying the characteristics (values, principles, and components) of Indigenous PHC service delivery models.

\section{Method}

In 2015, the Leadership Group as part of The Centre of Research Excellence in Aboriginal Chronic Disease Knowledge Translation and Exchange, identified the need to document the characteristics of Indigenous PHC service delivery models. Guided by the Leadership Group, a review team was formed comprising one Aboriginal [SH] and three non-Indigenous researchers [CD, AM, ZM]. A key feature of this review was the combination of perspective and skills that the Leadership Group and the researchers brought to the project. This included expertise in systematic and scoping reviews as well as an understanding of Indigenous beliefs, values and experiences.

A scoping review methodology was chosen, as it is the most appropriate methodology for synthesising a body of evidence that has yet to be comprehensively reviewed [19]. Additionally, scoping review methodology is acknowledged as appropriate method to identify concepts or characteristics in the literature [19], such as the characteristics of Indigenous PHC service delivery models. This systematic scoping review followed the Joanna Briggs Institute Scoping Review Methodology [20]. The review team developed and published a protocol prior to commencing the systematic scoping review [21], that outlined the intended approach and method, which is summarised below.

\section{Inclusion criteria}

Concept - the characteristics (values, principles, and components) of service delivery models implemented within an Indigenous PHC service.

Context - PHC services that provided care predominantly for Indigenous peoples.

Indigenous peoples were defined as:

Indigenous populations are communities that live within, or are attached to, geographically distinct traditional habitats or ancestral territories, and who identify themselves as being part of a distinct cultural group, descended from groups present in the area before modern states were created and current borders defined. They generally maintain cultural and social identities, and social, economic, cultural and political institutions, separate from the mainstream or dominant society or culture ([22](para. 1)).

Primary health care was defined as: 
socially appropriate, universally accessible, scientifically sound first level care provided by health services and systems with a suitably trained workforce comprised of multi-disciplinary teams supported by integrated referral systems in a way that: gives priority to those most in need and addresses health inequalities; maximises community and individual self-reliance, participation and control; and involves collaboration and partnership with other sectors to promote public health. Comprehensive primary health care includes health promotion, illness prevention, treatment and care of the sick, community development, and advocacy and rehabilitation ([23] (para. 3)).

The above definitions ensured all reviewers shared the same understanding of Indigenous and PHC and all included studies met the inclusion criteria.

\section{Types of studies}

All study types and methods including grey (unpublished) literature published in English between September 1978 and May 2015 were considered. Given that the concept of PHC was broadly adopted in September 1978 [24], papers prior to this date were excluded.

\section{Search terms}

Aboriginal OR Aborigine OR Indigenous OR First Nation OR Maori OR Inuit OR American Indian OR Alaskan Native OR Native Hawaiian AND primary health care OR comprehensive primary health care OR medical service OR health service OR community care OR community health care AND model.

\section{Search strategy}

An initial search of PubMed was conducted to identify text words contained in the title and abstract as well as any index terms that could be used as alternate search terms. A second, more detailed search was then undertaken using the identified search terms across PubMed, EBSCO CINAHL, Embase, Informit, Mednar, and Trove. The detailed search strategy used for PubMed, which is the basis for all other databases searched can be found in Additional file 1. The reference list of all identified studies were also hand-searched for additional studies which met the inclusion criteria. As a final step, a post was placed on ResearchGate to identify any additional literature (particularly grey literature) which may not have been widely available through conventional databases.

\section{Study selection}

The selection of studies was performed by four of the authors [SH, CD, AM, ZM] over two stages - title and abstract review; and full text review, against the inclusion criteria. During the two stages of study selection, author one $[\mathrm{SH}]$ reviewed all studies and this was checked by either one of the other three authors [CD, $\mathrm{AM}, \mathrm{ZM}]$. Any disagreements were discussed and resolved by authors.

\section{Charting of data}

All papers were imported into QSR International's NVivo 10 software [25] for extraction of reported characteristics. This analysis was initially conducted independently by the four authors [SH, CD, AM, ZM]; $[\mathrm{SH}]$ checked the data extraction and analysis of the three other authors [CD, AM, ZM], while a combination of the other authors checked the data extraction and analysis initially conducted by [SH]. Any disagreements were discussed and resolved between the four authors. Thematic analysis was used to group the extracted findings into characteristics. Findings were reviewed by members of the Leadership Group on two separate occasions during the synthesis process in order to provide an Aboriginal and Torres Strait Islander perspective on their validity.

\section{Results}

The original search identified 2599 studies (Fig. 1), from which 402 duplicates were removed, leaving 2197 studies for screening of title and abstract against the inclusion criteria. From this we retrieved 141 studies for full text review, of these 62 met the inclusion criteria (Additional file 2). Study selection follows the PRISMA reporting guidelines for study selection [26].

\section{Description of studies}

The majority of studies included in the review were conducted in Australia [27-58], 18 studies were conducted in the United States [59-76], five in Canada [77-81], four studies in New Zealand [82-85] and one study each in Papua New Guinea [86], Mexico [87] and Peru [88]. Of the studies included, the majority of service delivery models focused on PHC service delivery more generally [29-32, 34, 36, 40-42, $49,52-54,56,58,61,64,66,67,69,71,73,75,76$, 81, 82, 84-86]; while the others studies focused on specific areas such as women's, maternal and infant health $[27,45,47,57,60,78,79,87,88]$; mental health $[70,78,80]$; oral health $[33,39,65]$; eye health [62, 77]; adult health [38]; prevention and health promotion [63, 72, 74]; public and environmental health [59]; homecare [68]; prison health [50, 51, 55]; asthma [35, 37]; diabetes [83]; alcohol and other drugs [46]; medicine access [89]; and continuous quality improvement $[28,43]$. 


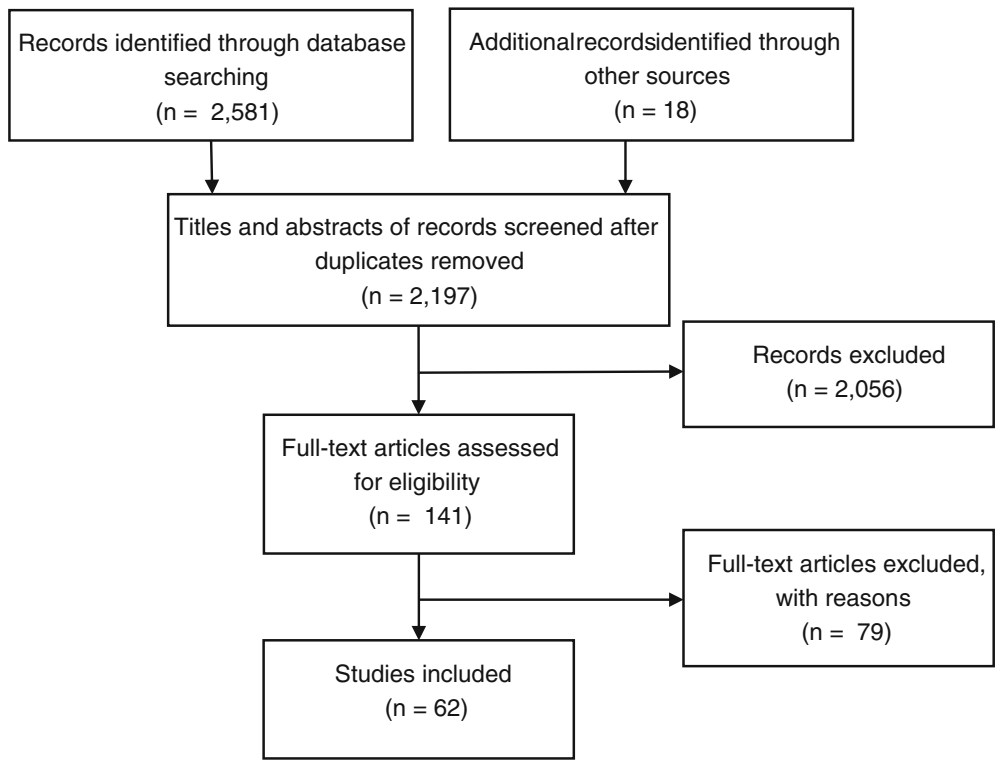

Fig. 1 Flow chart of study selection for scoping review process

\section{Characteristics}

Of the included studies, eight characteristics of Indigenous PHC service delivery models were identified - accessible health services, community participation, continuous quality improvement, culturally appropriate and skilled workforce, culture, flexible approach to care, holistic health care, and self-determination and empowerment. These characteristics underpin many of the service delivery models in this review.

While culture was initially thought to be one of the eight characteristics identified, it became evident through the process of thematic analysis, that it played a central role and was embedded throughout all Indigenous PHC service delivery models. Although the studies did not provide a definition of culture in the context of Indigenous PHC services, they did however demonstrate how aspects of culture were embedded within services and how culture is respected.

Pivotal strategies for embedding culture included the incorporation of local Indigenous cultural values [30, 54, 69, 73, 82]; customs and beliefs $[38,53,75,77,79,80$, $82,85,88]$, as well as traditional healing and practices $[36,77,79]$ into the service delivery model. Focusing on the needs of the individual and on the health and wellbeing of their families and communities [33, 34, 85]; respecting women's and men's cultural needs [38, 41, 45, 72 , 87], such as women only discussing women's health business with other women [45] or gender specific services and programs [41, 72, 87]; and ensuring the local communities were engaged with $[40,85]$ and in control of, Indigenous health services, $[52,79,85]$ were ways in which culture was embedded into service delivery models and ensured the delivery of culturally appropriate care and made services more acceptable to Indigenous communities. Other practical examples of how culturally appropriate service delivery was achieved, included creating welcoming [41] and comfortable spaces [72], and family-friendly environments [48, 85], through to for example, the use of Indigenous artwork and Indigenous signage [27], and developing culturally appropriate prevention and health promotion resources $[37,50,63,72]$.

Culture was strengthened in many instances by ensuring local languages were spoken within the service $[38,41,61,72,77,80,87,88]$. This was often achieved through the employment of local Indigenous staff who also acted as interpreters for nonIndigenous health staff [81]. The employment of local Indigenous staff also incorporated aspects of cultural mentoring [38, 45, 81] ensuring non-Indigenous staff were culturally competent $[45,84]$ and aware of local protocols and values [58]. This contributed to ensuring cultural safety, a concept which extended beyond being simply aware of cultural differences to incorporating a deeper level of interaction and thoughtful practice, as defined by those who receive services [38, $47,48,50,52,56,66,79]$.

The other seven characteristics of Indigenous PHC service delivery models are described in Table 1 .

While each of these characteristics were clearly identifiable as independent themes within the literature, the interdependence between characteristics was also evident. As mentioned, culture was interwoven throughout the seven other characteristics. Culture facilitated 
Table 1 Characteristics of indigenous PHC service delivery models

\section{Accessible Health Services}

- providing affordable health care $[61,87]$ at either no cost or low cost [85].

- ensuring a broad range of services are available [50], in a variety of locations and settings $[29,30,37,41,47,49,56,71-73,78,80-82,84$, 87], including increased opening hours, walk in appointments $[41,47,52,60,68,76]$ and transport $[47,60,72]$.

- delivering acceptable care which focused on building trust with communities [87] ensuring patients felt supported [38] providing assurances in relation to privacy and confidentiality [38,80], and implementing services underpinned by cultural respect, social justice and equality [47].

- ensuring awareness in the sense that communities know the service exists by providing outreach and mobile services [82], participating in community events and holding screening days [38], encouraging patients to share their positive experiences and promote the service to others [71].

\section{Community Participation}

- ensuring Indigenous ownership of health services which enables Indigenous peoples to own and manage their health service [85], and ensures the service is accountable to the community [54]. - establishing Indigenous governance [30-32, 34, 35, 40, 60, 61, 64, $70,82]$ including members from local community on governing boards, in order to encourage community involvement and ownership, while at the same time building capacity within local communities $[41,50,53]$.

- facilitating community consultation, engagement and collaboration in order to establish a strong relationship with the community $[32,84]$ and facilitate sharing of information $[83,88]$ ensuring that programs were culturally appropriate, accessible, engaging and empowering, and designed to take account of the local context and needs [34, 47, 54, 58, 74, 80, 82, 87].

- respecting the role and status of elders [36] and facilitating their involvement in the work and governance of services $[45,63,66]$.

\section{Continuous Quality Improvement}

- collecting and utilising data not simply to improve health outcomes but also meet the needs of each community by undertaking program evaluation [29], participating in quality improvement initiatives [28], reporting on performance [43], identifying clients who require a specific service, follow-up visits and client tracking [62], service planning and implementation [42, 64, 82], and service impact [42].

- evaluating services to measure health benefits for the community [54], assessing economic outcomes, baseline demographics in relation to increased service utilisation, health assessments and chronic disease care plans [42].

- undertaking research to strengthen health systems in order to meet the needs of the community with an emphasis on translating research findings into practice [28].

- establishing quality improvement processes with a focus on chronic conditions, monitoring health programs as well as management and follow-up care [43, 62], and community involvement in developing indicators [64], that also focus on cultural aspects of care, traditional approaches and receiving care in language [80].

\section{Culturally Appropriate and Skilled Workforce}

- employing a range of skilled staff both health and non-health personnel who are able to meet the needs of the local community $[30,36,41,49,50]$.

- establishing an Indigenous workforce comprising Indigenous Health Workers, mental health workers, social workers, nurses, doctors, administration staff, managers and traditional healers, all who are central to the delivery of services, providing a diverse range of care $[37,38,41,47,50,78,81,88]$, and within some service models, conducting the majority of the clinical work with clients $[31,38]$.
Table 1 Characteristics of indigenous PHC service delivery models (Continued)

- recognising that Indigenous staff often have responsibilities and obligations in relation to family and community, which were often conducted within the health service [54], such as interpreting and acting as mediators [78].

- providing training and development opportunities for all staff $[30,32,41,47,49,50,61,64,70,74,88]$, such as cultural awareness training for non-Indigenous staff $[38,58,81]$, and comprehensive training for staff working in remote areas or in isolation who require additional skills and knowledge to deal with an array of more com plex needs [61].

- training Indigenous Health Workers $[30,32,49,50,61,70]$ as a capacity building exercise [88], including more specialised health care such as dental [33, 39] or maternal health [47].

- recognising the need to build and grow the Indigenous workforce of the future by establishing long term strategies to mentor and recruit Indigenous students into health careers [80], leading to Indigenous staff going on to further training or study [32].

Flexible Approaches to Care

- tailoring approaches to identify $[36,50,55,66,72]$ and meet the needs of the local community $[29,38,41,47,48,50,52,54,58,64$, $69,70,83,87]$, and delivering a range of services [29], that are relevant, culturally appropriate and effective $[36,82]$.

- integrating health care services $[38,69,76,78,80,84]$, with a multidisciplinary team approach $[34,49,62,67-69,76]$, case management $[33,46,67,72,76,80]$, and continuity of care $[47,48,53,56,67,68,81,84]$.

- partnering and linking with other services to promote integration and cooperation between all support services, providing holistic care through a social view of health [39, 41, 47, 51, 80].

\section{Holistic Health Care}

- providing comprehensive primary health care, which is holistic $[32,34,37,80,82]$, supports the health and wellbeing of not only the individual but also their family and community [34, 49-51, 54, $66,69,73,85]$, and includes mental, emotional and spiritual needs alongside physical wellbeing $[57,60,80,84]$.

- offering a diverse range of services to clients [57], such as prevention and health promotion through to chronic disease care $[29,31,34,41,53,57,62,71]$, maternal and child health $[34,41,49$, $56,57,59,82]$, oral health $[39,42,50,53,57,64,65,82]$, ear health $[53,56,64]$ sexual health $[53]$, mental and social health $[34,41,56$, $57,59,78]$, alcohol and other drugs treatment $[50,53,82]$, pharmaceutical services $[44,56,69,78,80]$, aged care $[29,36,41]$ and disability services $[41,82]$.

- including prevention and health promotion initiatives developed and tailored to the needs of the local community, including general screening programs $[29,38,63,72,77,82]$ healthy lifestyles programs [56] needle exchange programs [29, 33, 56, 82], women's and men's health programs $[29,34]$, healthy eating, exercise and smoking cessation programs $[31,32,49,52,56,71,77]$, oral health [65], injury prevention [74], and supporting people to manage their own health [71].

- improving health literacy particularly in relation to early warning signs of suicide [70], increasing HIV/AIDs awareness [32], providing information about the harm that comes from alcohol, tobacco and other drugs [73], understanding food labelling [83], maintaining health and ensuring that people could detect early warning signs and understanding when to seek health care advice $[71,83]$.

- providing traditional healing as one option within the health service $[36,52,59,63,64,70,72,73,77,79-82,84-88]$.

- advocating for clients $[29,52]$, especially in relation to clients moving between other primary, secondary, tertiary, and essential non-health services $[60,84]$.

- engaging with the social determinants of health by supporting clients in accessing housing, employment, education, social security payments, and supporting people through the justice system $[29,41,49-51,53,56,57,59,73,80,82]$, 
Table 1 Characteristics of indigenous PHC service delivery models (Continued)

specifically for or customised to meet the needs of the community they served

$[32,47,84,88]$.

- providing advice in relation to public health initiatives not within the normal scope of mainstream PHC including sanitation system construction and maintenance, disease surveillance, environmental health, food distribution, and transportation [59].

- collaborating with other organisations such as schools, youth groups, prisons, disability and aged care services [29, 32, 81], and with councils, liquor outlets and grocers to reduce the supply of harmful products while increasing the availability of healthy options $[29,36,47,61]$.

\section{Self-determination and Empowerment}

- facilitating self-determination and empowerment of Indigenous communities in order to establish and manage their own Indigenous health services [30, 32], enabling clients to take control of their own health, at an individual and family level [69], building resilience [53], and enabling empowerment of clients through program engagement $[34,40,47,59,74,77,83,87]$.

- providing employment and training to promote the development of the local Indigenous health workforce, and build the capacity of the community $[32,40,41,87]$.

- facilitating leadership opportunities to ensure that Indigenous health care staff take on leadership roles within their communities [32] and provide positive role models for other Indigenous peoples $[32,70]$.

- promoting community development through the organisation of activities beyond health care [36, 49], such as cultural days and camps, reconciliation events and other community activities enable communities to draw on culture, increase social connectedness, and have pride in their identity [41].

assessable health services, informing the delivery of culturally appropriate services by making them acceptable by the community $[34,47,54,58,74,80,82,87]$. Culture was critical to ensuring community participation, enabling Indigenous ownership and governance by engaging communities [30-32, 34, 35, 40, 60, 61, 64, 70, 82], and engaging in quality improvement process and defining outcomes and indicators [64]. Culture was important in ensuring the approach to care is culturally appropriate and relevant [36, 82, 83], and holistic including comprehensive and providing a diverse range of care, which included traditional healing [36, 52, 59, 63, 64, 70, 72, 73, 77, 79-82, 84-88]. Culture informed and supported the philosophy underpinning Indigenous self-determination, particularly community participation, ensuring Indigenous peoples having the right and determination to decide how their PHC services should, and can be delivered [30, 33, 40, 49, 59, 69, 73, 82, 85].

Another example of interdependence between characteristics is culturally appropriate and skilled workforce, which was an enabler for culture, holistic health care and accessible health services. Employing local Indigenous staff helped to embed community cultural values, customs and beliefs into service delivery $[45,77,78,81]$. Workforce was central to the delivery of services, providing a holistic comprehensive $\mathrm{PHC}$ and a diverse range of care $[38,52,83,85,87,88]$. A culturally appropriate and skilled workforce also enabled services to be accessible and acceptable, by building trust with communities [45, 83, 87], ensuring patients felt supported [38, 41], providing assurances in relation to privacy and confidentiality [38, 80], and implementing services underpinned by cultural respect, social justice and equality [47].

As a result of identifying the characteristics of Indigenous PHC service delivery models, we have identified and described a new Indigenous PHC Service Delivery Model, as depicted in Fig. 2. Fundamental to this model is culture, as previously discussed culture plays a central role in Indigenous PHC service delivery models; and is encompassed by the seven other characteristics - accessible health services, community participation, continuous quality improvement, culturally appropriate and skilled workforce, flexible approach to care, holistic health care, and self-determination and empowerment. While Table 1 provides details as to how the characteristics have been embedded throughout other Indigenous PHC service delivery models, they also act as examples of how the characteristics of the Indigenous PHC Service Delivery Model can be implemented within other services.

\section{Discussion}

The aim of this scoping review was to identify the characteristics of Indigenous PHC service delivery models. We found that culture underpinned all aspects of the Indigenous PHC service delivery models identified in this review. In addition, we identified seven other distinct characteristics of Indigenous PHC service delivery models - accessible health services, community participation, continuous

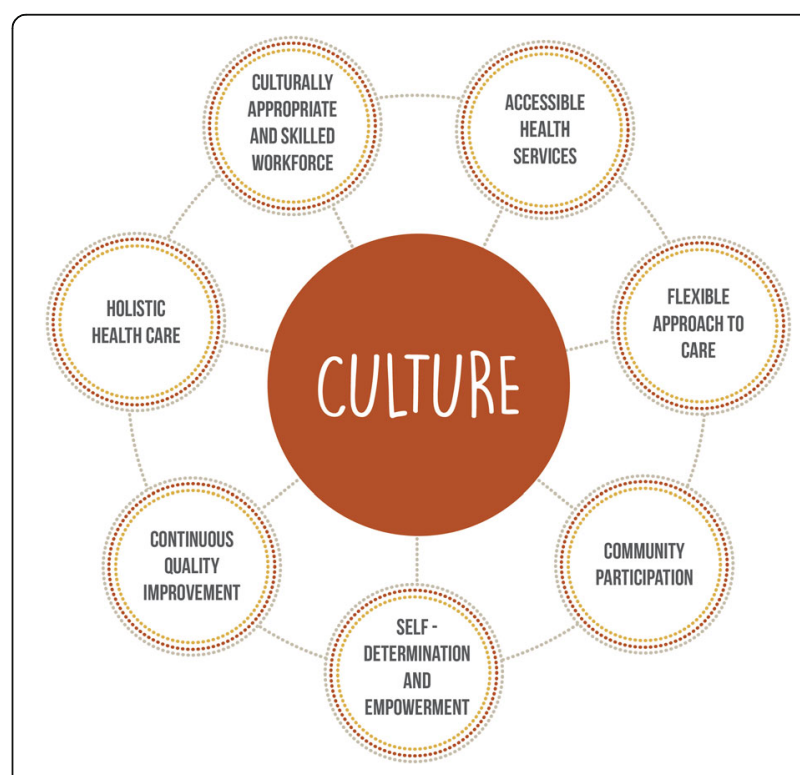

Fig. 2 Characteristics of Indigenous Primary Health Care Service Delivery Model 
quality improvement, culturally appropriate and skilled workforce, flexible approach to care, holistic health care, and self-determination and empowerment.

These findings suggest that Indigenous PHC service delivery models are somewhat different to many of the models of care developed within western contexts. For example, the Chronic Care Model [90] focuses on implementing evidence based care, mobilising community resources, enabling patient's self-management, and ensuring coordinated care and health promotion. The explicit role of culture in the provision of services is notably absent from this model. Culture is also notably absent from the World Health Organisation Innovative Care for Chronic Conditions Framework [91] and the Southgate Model of Comprehensive Primary Health Care in Australia model [92].

By contrast, it is evident that local cultural values, customs and beliefs were at the centre of and underpinned all aspects of care in Indigenous PHC service delivery models. This was a common thread in the majority of the studies included in this scoping review. The role of culture as a defining characteristic, therefore, provides the greatest distinction between Indigenous PHC service delivery and other models of care. This is consistent with the growing literature on culture and health, which describes the importance of culture and its effect on health and wellbeing [2, 93-95], including in nonIndigenous populations [96]. These are potential lessons mainstream health care services could learn from in order to make their services more culturally safe and appropriate to Indigenous peoples. Three characteristics stand out as distinctive aspects of Indigenous PHC service delivery models. These are culturally appropriate and skilled workforce; community participation; and self-determination and empowerment. At the heart of a culturally appropriate and skilled workforce were the Indigenous staff. This supports the belief that Indigenous PHC services are best delivered by Indigenous peoples [17]. In particular, employing local Indigenous staff helps to embed the community's cultural values, customs and beliefs into service delivery. Indigenous peoples providing health care to their own people has been shown to improve health outcomes related to diabetes [97], asthma [98], mental health and maternal and infant care [99]. Importantly, local Indigenous staff provide more acceptable care [100], as well as encouraging access to PHC more generally [101]. One of the many challenges faced by Indigenous PHC services is the need to maintain current levels of Indigenous staff, while at the same time, growing their Indigenous health workforce [66, 80]. This requires a partnership between Indigenous $\mathrm{PHC}$ services and governments to ensure the growth of the sector is done in a way that is meaningful and culturally safe.
Community participation was found to be particularly important for ensuring Indigenous $\mathrm{PHC}$ services continue to identify, understand and address the needs of local Indigenous peoples. Community participation also facilitated Indigenous governance and ownership. One example is the Southcentral Foundation in Anchorage Alaska, it is possibly one of the most well-known Indigenous models of care (four of the included studies are from the Southcentral foundation $[60,64,69,76])$. The success of this model, including significant improvements in health outcomes is associated with the notion of 'customer-owners' ([102] p. 1). The model ensures that Alaska Native people are in control of their health service and the relationship that is built and maintained by the service with its 'customer-owners' ([102] p. 1). Many of these services included in this review were also underpinned by the philosophy that Indigenous peoples having the right to decide how their PHC services should be developed and delivered. Aboriginal Community Controlled Health Organisations in Australia have been identified as exemplars of these types of community governance models [103], further demonstrating the link between community control and positive health outcomes.

Self-determination and empowerment were the driving principles behind the establishment of many Indigenous $\mathrm{PHC}$ services included in this review. Indigenous PHC services facilitated a number of opportunities for selfdetermination and empowerment. These include ensuring that Indigenous peoples are able to take control of their own health service; the employment and training of Indigenous peoples; and just as importantly community development initiatives such as cultural days and camps, and reconciliation events. Previous studies have demonstrated an association between empowerment of Indigenous peoples and communities and better health outcomes for Indigenous peoples [104, 105]. As on example, a systematic review conducted by Minichiello et al. [106], found that tobacco intervention programs which had elements of self-determination and were relevant to the community were more likely to lead to positive outcomes such as reduced initiation and consumption of tobacco. Often self-determination and empowerment are associated with community participation. Community participation and mobilization in health care is essential for ensuring services can identify health needs and set priorities, plan, implement, monitor and evaluate services and programs, this is consistent with existing literature on community participation in health [107].

Indigenous PHC service delivery models are exceptional models of PHC, delivering health care to Indigenous peoples and communities across the globe, often to isolated populations or communities where no other 
service delivery model is viable. While there are numerous benefits to Indigenous PHC service delivery models, there are also limitations: Indigenous PHC service delivery models do not align with government funding mechanism [108, 109]; there is often a lack of funding to support specific Indigenous PHC services [108-110]; the need for services outweighs funding and the availability of services [108-110]; and the delivery of health care to Indigenous peoples is more expensive [108, 109].

Policies need to acknowledge and take into account the differences between Indigenous PHC models of care and other PHC models of care. This is particularly crucial when it comes to the funding of Indigenous PHC services, as the lack of sufficient funding together with the uncertainty that comes from short-term funding models led to the inability of services included in this review to support the unique characteristics of Indigenous PHC services [27, 32, 33, 40, 51, 54, 78, 82]. Funding models are one of the key drivers for how care is provided [111]. Yet it is also the case that until Indigenous PHC service delivery models are clearly articulated, policy makers will not be able to design appropriate funding mechanisms to support the way in which they provide care. We believe that the Indigenous PHC service delivery characteristics identified by this scoping review is one step towards making Indigenous PHC service delivery models explicit.

While this is the first review of its type to identify the characteristics of Indigenous PHC service delivery models, a review by Lewis and Myhra on Integrated care with Indigenous populations: a systematic review of the literature [112], was identified. However, that review focused on assessing how health care services are conceptualising and enacting integrated care with American Indian and First Nations populations in the United States and Canada and the successes and challenges of carrying out these interventions with this population. It identified the motivations for integration and its effectiveness. Our review was more inclusive of health services and Indigenous populations from across the global and focused on the characteristics of Indigenous PHC service delivery models rather than the motivations of one particular service delivery model.

\section{Conclusion}

Indigenous PHC services evolved as a result of mainstream health services inability to meet the needs of Indigenous peoples $[3,7,8]$ and Indigenous peoples often being excluded and marginalised from mainstream health services [9]. In addition, Indigenous communities wanted to be able to provide care to their communities that is culturally appropriate, comprehensive, holistic, accessible, and community controlled. The review identified eight characteristics of Indigenous PHC service delivery models. These characteristics were found to be global in their application, and provide insight and guidance to communities and organisations wishing to initiate an Indigenous PHC service and programs. The review also affirms and supports the philosophy underpinning Indigenous self-determination, particularly Indigenous peoples having the right and determination to decide how their PHC services should be and can be delivered for themselves. If communities and governments are genuinely serious about closing the gaps in life expectancy and morbidity between Indigenous and nonIndigenous peoples, then Indigenous PHC services must continue to be supported.

\section{Additional files}

Additional file 1: Search strategy. (DOCX $13 \mathrm{~kb}$ )

Additional file 2: Included studies. (DOCX $16 \mathrm{~kb}$ )

Abbreviations

PHC: Primary Health Care

\section{Acknowledgements}

The authors would like to thank the Centre of Research Excellence in Aboriginal Chronic Disease Knowledge Translation and Exchange Leadership Group for their guidance and support; Assoc Prof James Ward (Infection and Immunity, South Australian Health and Medical Research Institute), Dr. Odette Gibson (Wardliparingga Research Unit, South Australian Health and Medical Research Institute) and Prof Annette Braunack-Mayer (School of Public Health, The University of Adelaide) for their comments on the final draft; and Eliza Cobb, (Marketing and Communications, South Australian Health and Medical Research Institute) for your graphic design skills.

\section{Funding}

This research was supported by National Health and Medical Research Council (NHMRC No 1061242).

\section{Availability of data and materials \\ Not applicable.}

\section{Authors' contributions}

$\mathrm{NB}, \mathrm{AB}$ contributed to the conceptualisation of the review. $\mathrm{SH}, \mathrm{CD}, \mathrm{AM}$ and ZM designed the search strategy, were involved with study selection and review, analysis of data and drafting of manuscript. SH conducted the search. All authors reviewed and approved the final manuscript.

Ethics approval and consent to participate Not applicable.

\section{Consent for publication}

Not applicable.

\section{Competing interests}

The authors declare that they have no competing interests.

\section{Publisher's Note}

Springer Nature remains neutral with regard to jurisdictional claims in published maps and institutional affiliations.

\section{Author details}

'Wardliparingga Aboriginal Health Research Unit, South Australian Health and Medical Research Institute, Adelaide, South Australia, Australia. ${ }^{2}$ School of Public Health, Faculty of Health Sciences, The University of Adelaide, Adelaide, South Australia, Australia. ${ }^{3}$ Joanna Briggs Institute, Faculty of Health 
Sciences, The University of Adelaide, Adelaide, South Australia, Australia. ${ }^{4}$ Sansom Institute for Health Research, University of South Australia, Adelaide, South Australia, Australia. ${ }^{5}$ School of Education and School of Medicine, University of Wollongong, Wollongong, NSW, Australia.

\section{Received: 8 October 2017 Accepted: 16 January 2018}

\section{Published online: 25 January 2018}

\section{References}

1. Stephens C, et al. Indigenous peoples' health-why are they behind everyone, everywhere? Lancet. 2005;366(9479):10-3.

2. Gracey $M$, King M. Indigenous health part 1: determinants and disease patterns. Lancet. 2009:374(9683):65-75.

3. Australian Institute of Health and Welfare. Australia's health 2014. 2014 [14/ 04/2016]; Available from: https://www.aihw.gov.au/reports/australias-health/ australias-health-2014/contents/table-of-contents.

4. Statistics New Zealand. New Zealand period life tables: 2010-12. 2013 [14/ 04/2016]. Available from: http://www.stats.govt.nz/browse_for_stats/health/ life_expectancy/NZLifeTables_HOTP10-12.aspx.

5. Health Canada. Aboriginal health. 2005 [12/06/2016]; Available from: http:// www.hc-sc.gc.ca/hcs-sss/pubs/system-regime/2005-blueprint-plan-aborauto/fs-fi-03-eng.php.

6. Indian Health Service. Disparities 2015 [14/04/2016]; Available from: http:// www.ihs.gov/newsroom/factsheets/disparities/.

7. Ellison-Loschmann L, Pearce N. Improving access to health care among New Zealand's Maori population. Am J Public Health. 2006:96(4):612-7.

8. Lurie N. Mesauring disparities in access to care. In: Swift EK, editor. Guidnace for the national healthcare disparities report. Washington, D. C: The National Academies Press; 2002. p. 99-148.

9. Aboriginal Medical Service Redfern. Our history 2015 [10/08/2016]; Available from: http://amsredfern.org.au/dental-specialist-clinics-3/.

10. National Aboriginal Community Controlled Health Organisation. About NACCHO. 2016 14/07/2016]; Available from: http://www.naccho.org.au/ about/

11. Health Canada. Ten years of health transfer first nation and Inuit control. 2013 11/03/2016]; Available from: http://www.hc-sc.gc.ca/fniah-spnia/pubs/ finance/_agree-accord/10_years_ans_trans/index-eng.php.

12. Lavoie J. Policy and financing of indigenous primary health care providers in Canada, Australia and New Zealand. London, England: London School of Hygiene and Tropical Medicine; 2005

13. First Nations Health Authority. About us. 2015 13/05/2016]; Available from: http://www.fnha.ca/about.

14. Indian Health Service. Agency overview. 10/04/2016]; Available from: http:// www.ihs.gov/aboutihs/overview/.

15. Kunitz SJ. The history and politics of US health care policy for American Indians and Alaskan natives. Am J Public Health. 1996:86(10):1464-73.

16. Bergman $A B$, et al. A political history of the Indian Health Service. Milbank Q. 1999:77(4):571-604

17. National Aboriginal Community Controlled Health Organisation. Definitions. 2014 12/06/2016]; Available from: http://www.naccho.org.au/aboriginalhealth/definitions/.

18. National Aboriginal Community Controlled Health Organisation. NACCHO Vision 2014 12/06/2016]; Available from: http://www.naccho.org.au/aboutnacho/naccho-vision/.

19. Peters $M$, et al. Guidance for conducting systematic scoping reviews. International Journal of Evidence-Based Healthcare. 2015;13(3):141-6.

20. Peters $\mathrm{M}$, et al. Methodology for JBI scoping reviews. In: Aromataris $\mathrm{E}$, editor. The Joanna Briggs institute reviewers' manual 2015. Adelaide: The Joanna Briggs Institute; 2015.

21. Harfield S, Davy C, Kite E, McArthur A, Munn Z, Brown N, et al. Characteristics of Indigenous primary health care models of service delivery: a scoping review protocol. JBI Database System Rev Implement Rep. 2015;13(11):43-51.

22. World Health Organisation. Indingenous populations. 2015 [04/06/2016]; Available from: http://www.who.int/topics/health_services_indigenous/en/

23. Thomas SL, Wakerman J, Humphreys JS. What core primary health care services should be available to Australians living in rural and remote communities? BMC Family Pract. 2014;15:143.

24. World Health Organisation. Declaration of Alma-Ata. International conference on primary health care 1978 5-12 September 1978 [14/04/2016]; Available from: http://www.who.int/publications/almaata_declaration_en.pdf.

25. QSR International Pty Ltd, NVivo qualitative data analysis software. 2012.
26. Moher D, et al. Preferred reporting items for systematic reviews and metaanalyses: the PRISMA statement. PLoS Med. 2009;6(7):e1000097.

27. The Dreamers - Art Therapy for Women. Aborig Islander Health Worker J. 2002;26(6):10

28. Bailie R, et al. A systems-based partnership learning model for strengthening primary healthcare. Implement Science. 2013:8:143.

29. Bartlett B, Boffa J. Aboriginal community controlled comprehensive primary health care: the central Australian aboriginal congress. Australian Journal of Primary Health. 2001;7(3):74-82

30. Bennett M. An aboriginal model of care. Nurs Times. 1988;84(19):56-8.

31. Birks $M$, et al. Models of health service delivery in remote or isolated areas of Queensland: a multiple case study. Aust J Adv Nurs. 2010;28(1):25-34

32. Campbell D, Ellis R. Models of excellence in indigenous community health part one: the aboriginal medical service, Redfern. Aboriginal and Islander Health Worker Journal. 1995;19(4):4-14.

33. Campbell MA, et al. The oral health care experiences of NSW aboriginal community controlled health services. Aust N Z J Public Health. 2015:39(1):21-5.

34. Central Australian Aboriginal Congress. Congress logic model. Alice Springs: Central Australian Aboriginal Congress.

35. Chang $A B$, et al. Asthma management in indigenous children of a remote community using an indigenous health model. J Paediatr Child Health. 2000;36(3):249-51

36. Copeman RC. Assessment of aboriginal health services. Community Health Studies. 1988;12(3):251-5

37. Dawson A, Russel A, Caponi A. Asthma project at Pika Wiya health services: identifying barriers and developing resources. Aboriginal and Islander Health Worker Journal. 2003;27(5):20-2.

38. DiGiacomo $\mathrm{M}$, et al. Facilitating uptake of aboriginal adult health checks through community engagement and health promotion. Qual Prim Care. 2010:18(1):57-64

39. Dyson K, Kruger E, Tennant M. Networked remote area dental services: a viable, sustainable approach to oral health care in challenging environments. Australian Journal of Primary Health. 2012;20(6):334-8.

40. Freeman $T$, et al. Service providers' views of community participation at six Australian primary healthcare services: scope for empowerment and challenges to implementation. Int J Health Plann Manag. 2014;

41. Freeman $T$, et al. Cultural respect strategies in Australian aboriginal primary health care services: beyond education and training of practitioners. Aust N Z J Public Health. 2014;38(4):355-61.

42. Gajjar D, et al. A case study in the use of evidence in a changing political context: an aboriginal and Torres Strait islander health service re-examines practice models, governance and financing. Aust Health Rev. 2014;38(4): 383-6.

43. Gardner $\mathrm{KL}$, et al. Understanding uptake of continuous quality improvement in indigenous primary health care: lessons from a multi-site case study of the audit and best practice for chronic disease project. Implement Science. 2010:5:21

44. Kelaher $\mathrm{M}$, et al. Improving access to medicines among clients of remote area aboriginal and Torres Strait islander health services. Aust N Z J Public Health. 2006;30(2):177-83.

45. Kelly J, Luxford Y. Yaitya tirka madlanna warratinna: exploring what sexual health nurses need to know and do in order to meet the sexual health needs of young aboriginal women in Adelaide. Collegian. 2007:14(3):15-20.

46. Lovett $R$, et al. Walan Girri: developing a culturally mediated case management model for problematic alcohol use among urban indigenous people. Aust Health Rev. 2014;38(4):440-6.

47. Murphy $E$, Best $E$. The aboriginal maternal and infant health service: a decade of achievement in the health of women and babies in NSW. New South Wales Public Health Bulletin. 2012;23(3-4):68-72.

48. Panaretto $\mathrm{KS}$, et al. Impact of a collaborative shared antenatal care program for urban indigenous women: a prospective cohort study. Med J Aust. 2005 182(10):514-9.

49. Panaretto KS, et al. Aboriginal community controlled health services: leading the way in primary care. Med J Aust. 2014;200(11):649-52.

50. Poroch N, Winnunga Nimmityjah Aboriginal Health Service. You do the crime, you do the time : best practice model of holistic health service delivery for aboriginal and Torres Strait islander inmates of the ACT prison. Narrabundah, ACT: Winnunga Nimmityjah Aboriginal Health Service, xxviii; 2007. p. 260.

51. Poroch $\mathrm{N}$, et al. Aboriginal health workers at Winnunga Nimmityjah aboriginal health service caring for the needs of aboriginal people in the 
new act prison and the needs of their families. Aboriginal and Islander Health Worker Journal. 2012;36(1):6-8. 17

52. Reeve $\mathrm{C}$, et al. Strengthening primary health care: achieving health gains in a remote region of Australia. Med J Aust. 2015;202(9):483-8.

53. Stewart J, Raiwaqavuka T. Working with a community controlled health organization to develop an evidence base for their practice models. Australas Psychiatry. 2009;17(Suppl 1):S79-82.

54. Taylor J, et al. Contemporary management issues for aboriginal community controlled health services. Aust Health Rev. 2001;24(3):125-32.

55. Tongs J, Chatfield H, Arabena K. The Winnunga Nimmityjah aboriginal health service holistic health Care for Prison Model. Aboriginal and Islander Health Worker Journal. 2007;31(6):6-8.

56. Tongs J, Poroch N. Winnunga Nimmityjah aboriginal health service 19882014: breaking barriers in aboriginal research and services. Aust Aborig Stud. 2014(2):94-100.

57. Townsville Aboriginal Islanders Health Services Ltd. The mums and babies project. Families Australia Bulletin. 2003;3(Winter):10-1.

58. Wakerman J, Field P. Remote area health service delivery in Central Australia: primary health care and participatory management. Australian Journal of Primary Health. 1998;6(1):27-31.

59. Allison MT, Rivers PA, Fottler MD. Future public health delivery models for native American tribes. Public Health. 2007;121(4):296-307.

60. Baldwin M, Stevenson Y. A model for providing prenatal health care to indigenous women living in remote areas. International Journal of Circumpolar Health. 2001;60(4):623-31.

61. Berner BJ. Provision of health care in a frontier setting: an Alaskan perspective. Journal of the American Association of Nurse Practitioners. 1992;4(3):89-94

62. Bowyer NK. A primary care team approach to the prevention of ocular complications of diabetes: a program review. J Am Optom Assoc. 1997; 68(4):233-42.

63. Brant JM, Fallsdown D, Iverson ML. The evolution of a breast health program for plains Indian women. Oncol Nurs Forum. 1999;26(4):731-9.

64. Centre for Primary Care. Human Systems for Southcentral Foundation's Nuka system of care. Harvard: Boston, Massachusetts; 2015.

65. Chiarchiaro G. The Indian Health Service in Oklahoma: dental service for American Indians. Journal - Oklahoma Dental Association. 1997;87(3):24-8.

66. Coll KM, Mohatt G, LeMaster PL. Feasibility assessment of the service delivery model. Am Indian Alsk Native Ment Health Res. 2004;11(2):99-108.

67. Dietrich AJ, Olson AL. Model system of ongoing care for native Americans a 5-year followup. Public Health Rep. 1986;101(2):184-6.

68. Johnston JM, et al. Tribal implementation of a patient-centred medical home model in Alaska accompanied by decreased hospital use. International Journal of Circumpolar Health. 2013;72

69. Eby DK. Primary care at the Alaska native medical center: a fully deployed "new model" of primary care. International Journal of Circumpolar Health. 2007;66(Suppl 1):4-13.

70. Kahn MW, et al. An indigenous community mental health service on the Tohono O'odham (Papago) Indian reservation: seventeen years later. Am J Community Psychol. 1988;16(3):369-79.

71. Langwell K, et al. An American Indian community implements the chronic care model: evolution and lessons learned. Health Promot Pract. 2014;15(2): 23S-28.

72. Lantz PM, et al. Implementing women's cancer screening programs in American Indian and Alaska native populations. Health Care for Women International. 2003;24(8):674-96.

73. Nebelkopf E, King J. A holistic system of care for native Americans in an urban environment. J Psychoactive Drugs. 2003;35(1):43-52.

74. Smith RJ 3rd, Dellapenna AJ Jr, Berger LR. Training injury control practitioners: the Indian Health Service model. Futur Child. 2000;10(1):175-88

75. West EA. The cultural bridge model. Nurs Outlook. 1993;41(5):229-34.

76. Driscoll DL, et al. Process and outcomes of patient-centered medical care with Alaska native people at Southcentral foundation. Ann Fam Med. 2013; 11:S41-9.

77. Arora S, Kurji AK, Tennant MT. Dismantling sociocultural barriers to eye care with tele-ophthalmology: lessons from an Alberta Cree community. Clinical and Investigative Medicine. 2013;36(2):E57-63.

78. Auclair G, Sappa M. Mental health in Inuit youth from Nunavik: clinical considerations on a transcultural, interdisciplinary, community-oriented approach. Journal of the Canadian Academy of Child and Adolescent Psychiatry. 2012;21(2):124-6.
79. Benoit C, Carroll D, Chaudhry M. In search of a healing place: aboriginal women in Vancouver's downtown eastside. Soc Sci Med. 2003;56(4):821-33.

80. Maar MA, et al. Innovations on a shoestring: a study of a collaborative community-based aboriginal mental health service model in rural Canada. Int J Ment Heal Syst. 2009;3.

81. Tyree E. Culture care values, beliefs and practices observed in empowerment of American Indian community health representatives. Loyola University Chicago; 2007. p. 159. https://search.proquest.com/ openview/c343a6f2a0a16fc19940cc9457a5a008/1?pq-origsite=gscholar\&cbl= 18750\&diss $=y$.

82. Crengle $\mathrm{S}$. The development of Māori primary care services. Pacific Health Dialog. 2000;7(1):48-53.

83. Janssen J, Nelson K. Meeting the needs of Māori with diabetes: evaluation of a nurse-led service. Nurs Prax N Z. 2014;30(3):6-18.

84. Lyford S, Cook P. The Whanaungatanga model of care. Nurs Prax N Z. 2005; 21(2):26-36

85. Maniapoto T, Gribben B. Establishing a Māori case management clinic. N Z Med J. 2003;116(1169)

86. Davy CP, Patrickson M. Implementation of evidence-based healthcare in Papua New Guinea. International Journal of Evidence-Based Healthcare. 2012:10(4):361-8.

87. Pelcastre-Villafuerte $B$, et al. Community-based health care for indigenous women in Mexico: a qualitative evaluation. Int J Equity Health. 2014;13:2.

88. Gabrysch S, et al. Cultural adaptation of birthing services in rural Ayacucho, Peru. Bull World Health Organ. 2009;87:724-9.

89. Keleher $\mathrm{H}$. Why primary health care offers a more comprehensive approach to tackling health inequities than primary care. Australian Journal of Primary Health. 2001;7(2):57-61.

90. Wagner EH, Austin BT, Von Korff M. Organizing care for patients with chronic illness. Milbank Q. 1996;74(4):511-44.

91. World Health Organisation. Innovative care for chronic conditions: building blocks for action: global report. Geneva, Switzerland; 2002.

92. Lawless A, et al. Developing a good practice model to evaluate the effectiveness of comprehensive primary health care in local communities. BMC Fam Pract. 2014;15(1):1-9.

93. Anderson KM, Olson S. Leveraging culture to address health inequalities: examples from native communities. Seattle, United States of America: National Academies Press; 2013.

94. Morrissey M, et al. Culture as a determinant of aboriginal health. In: Anderson I, Baum F, Bentley M, editors. Beyond Bandaids: exploring the underlying social determinants of aboriginal health. Papers from the social determinants of aboriginal health workshop, Adelaide, July 2004. Darwin, Australia: Cooperative Research Centre for Aboriginal Health; 2007. p. 239-54.

95. National Advisory Committee on Health and Disability. The social, cultural and economic determinants of health in New Zealand: action to improve health. New Zealand: Wellington; 1998.

96. Napier AD, et al. Culture and health. Lancet. 2014;384(9954):1607-39.

97. Si D, et al. Aboriginal health workers and diabetes care in remote community health centres: a mixed method analysis. Med J Aust. 2006; 185(1):40-5

98. Chang $A B$, et al. Indigenous healthcare worker involvement for indigenous adults and children with asthma. The Cochrane Database of Systematic Reviews. 2010:5

99. Stamp G, et al. Aboriginal maternal and infant care workers: partners in caring for aboriginal mothers and babies. Rural Remote Health. 2008:8(3):883.

100. McGrath PD, et al. The case for aboriginal health workers in palliative care. Aust Health Rev. 2007:31(3):430-9.

101. Murray RB, Wronski I. When the tide goes out: health workforce in rural, remote and indigenous communities 1(2006):37. eLibrary. Web. 13 Apr. 2016. Med J Aust. 2006;185(1):37-8.

102. Gottlieb K. The Nuka system of care: improving health through ownership and relationships. International Journal of Circumpolar Health. 2013;72

103. Bath J, Wakerman J. Impact of community participation in primary health care: what is the evidence? Australian Journal of Primary Health. 2015;21(1):2-8.

104. Tsey $\mathrm{K}$, et al. Empowerment and indigenous Australian health: a synthesis of findings from family wellbeing formative research. Health \& Social Care in the Community. 2010;18(2):169-79.

105. Jersky $M$, et al. Improving health service access and wellbeing of young aboriginal parents in an urban setting: mixed methods evaluation of an arts-based program. Aust N Z J Public Health. 2016;40(S1):S115-21. 
106. Minichiello A, et al. Effective strategies to reduce commercial tobacco use in indigenous communities globally: a systematic review. BMC Public Health. 2016;16(1):1-25.

107. Sacks $E$, et al. Community involvement in health systems strengthening to improve Global Health outcomes: a review of guidelines and potential roles. International Quarterly of Community Health Education. 2017;37(3-4):139-49.

108. Alford K. Economic value of aboriginal community controlled health services. Canberra, Australia: National Aboriginal Community Controlled Health Organisation; 2014.

109. Russell L, Smiler K, Stace H. Improving Māori health and reducing inequalities between Mãori and non-Mãori: has the primary health care strategy worked for Māori? New Zealand: Health Research Council Of New Zealand and The Ministry Of Health Wellington; 2013.

110. Boccuti C, Swoope C, Artiga S. The role of Medicare and the Indian Health Service for American Indians and Alaska natives: health, access and coverage: Kaiser Family Foundation; 2014.

111. McDonald J, et al. The implementation and impact of different funding initiatives on access to multidisciplinary primary health care and policy implications. Med J Aust. 2008;188(8 Suppl):S69-72.

112. Lewis ME, Myhra LL. Integrated care with indigenous populations: a systematic review of the literature. Am Indian Alsk Native Ment Health Res. 2017:24(3):88-110

\section{Submit your next manuscript to BioMed Central} and we will help you at every step:

- We accept pre-submission inquiries

- Our selector tool helps you to find the most relevant journal

- We provide round the clock customer support

- Convenient online submission

- Thorough peer review

- Inclusion in PubMed and all major indexing services

- Maximum visibility for your research

Submit your manuscript at www.biomedcentral.com/submit 